\title{
Simulation Studies on the Design of a Voltage-Controlled Oscillator
}

\author{
J.W. Ye, J.S. Chen \\ The PLA Computer Technology Resource Center at Huanan \\ Division \\ Guangzhou, China
}

\begin{abstract}
The location and fine adjustment of the center frequency as well as the fine adjustment of power in a resonance loop remain a challenge in the field of voltage-controlled oscillator (VCO) design. Firstly, when the conditions of stable oscillation and the VOC with certain types of tuning bandwidth are met, the appropriate circuit configuration needs to be identified. Specifically, in the circuit topology of the oscillator, the varactor-centered reactance compensation network should be connected to the base of the transistor in order to serve as a tuned network, with the output of the emitter followed. By using simulation analyses, the feedback between the electrodes is then achieved by terminating a section of open-circuit microstrip line to the collector. Moreover, the adjustment and variation of the feedback quantity between the electrodes, as well as the variation and fine adjustments of power and frequency are observed during the simulation process. As a result, several issues related to the location and adjustment of the parameters and center frequency in designing a complicated VCO circuit have been effectively settled.
\end{abstract}

Keywords-voltage-controlled oscillator; s-parameter; tuning sensitivity

\section{INTRODUCTION}

A microwave solid-state oscillator is regarded as the 'heart' of a microwave transmitter, which represents a crucial component of microwave communication systems. With the rapid development of semiconductor technology, the microwave solid-state oscillator has gained wide applications in the field of microwave relay communications, satellite communications, radar, remote control and microwave testing instruments, and its performance could directly and significantly affect the performance of the entire communication systems. Accordingly, the development of high-performance microwave oscillator plays an important role in improving the quality of communication lines and the reliability of communications.

\section{THE DESIGN OF THE VOLTAGE-CONTROLLED OSCILLATOR (VCO) AND RELATED ANALYSES}

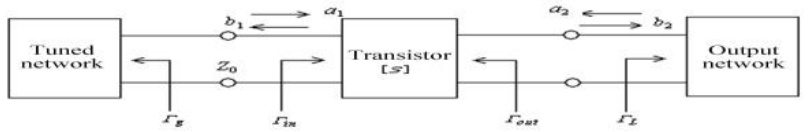

FIGURE I. PRINCIPLE OF A TWO-PORT OSCILLATOR.

The microwave transistor with certain configuration can be regarded as a two-port network, which can also become a core component of an oscillator under appropriate conditions.

\author{
J.F. Ye \\ School of Information Science and Technology, Donghua \\ University \\ Shanghai, China
}

According to its working mechanisms, the oscillator can be classified as either a feedback oscillator or a negative resistance oscillator, and the negative resistance is likely to be introduced to the circuit by the positive feedback. In doings so, the feedback oscillator could also be viewed, to some extent, as a negative resistance oscillator in essence. By virtue of the positive feedback of the capacitance between two electrodes in the device, the positive feedback of the external circuit, or the combined feedback network between the internal and external circuit, the negative resistance in a two-port oscillator could be formed. Fig. 1 displays the principle diagram of a two-port oscillator, where in the scattering matrix of the triode transistor $[S]$ is denoted by $S_{11}, S_{12}, S_{21}$ and $S_{22}$. Meanwhile, $\Gamma_{g}$ and $\Gamma_{L}$ denote the reflection coefficients of the tuned network and the output network, respectively.

According to the definition of the scattering parameters in a two-port network, the following expressions can be obtained:

$$
\begin{aligned}
& \Gamma_{\text {in }}=\frac{b_{1}}{a_{1}}=S_{11}+\frac{S_{12} S_{21} \Gamma_{L}}{1-S_{22} \Gamma_{L}} \\
& \Gamma_{\text {out }}=\frac{b_{2}}{a_{2}}=S_{22}+\frac{S_{12} S_{21} \Gamma_{g}}{1-S_{11} \Gamma_{g}}
\end{aligned}
$$

Moreover, the oscillating conditions can be described as:

$$
k=\frac{1-\left|S_{11}\right|^{2}-\left|S_{22}\right|^{2}+|D|^{2}}{2\left|S_{12} S_{21}\right|}<1, \Gamma_{g} \Gamma_{\text {in }}=1, \quad \Gamma_{L} \Gamma_{\text {out }}=1
$$

Where $D=S_{11} S_{22}-S_{12} S_{21}$.

Since the network consisting of passive devices is connected to both input and output ports, both $\left|\Gamma_{g}\right|$ and $\left|\Gamma_{L}\right|$ are less than 1 , which suggests that the oscillation should satisfy the following expressions, $\left|\Gamma_{i n}\right|>1$ and $\left|\Gamma_{\text {out }}\right|>1$. Furthermore, as long as one expression is true, then another one should be true as well. Generally speaking, the oscillating conditions can be determined by selecting one port with its modulus greater than 1 [1].

Unlike with the conventional oscillator, the VCO exhibit an instantaneous frequency, and is subjected to the effects of specific bias voltage. In a frequency synthesizer, the instantaneous frequency $\omega(t)$ is determined by the output error voltage of the loop filter $U_{\mathrm{d}}(t)$, i.e., $\omega(t)=\omega_{0}+K_{V} U_{\mathrm{d}}(t)$, where $\omega_{0}$ and $K{ }_{V}$ denote the center frequency and the voltage-controlled sensitivity, whereas $K_{V}$ denotes the 
variation of the angular frequency of the VCO induced by the unit control voltage.

Fig. 2 illustrates the circuit configuration of the VCO. With respect to the oscillation tube $T_{2}$, the low-power-consumption N-P-N bipolar silicon transistor AT 41511 manufactured by HP is selected. As a plastic-package surface mount device (SMD), AT 41511 has been commonly used in the low-noise very-high-frequency (VHF) and ultra-high-frequency (UHF) microwave wide-band amplifiers and oscillators. The primary performance parameters of the system are listed below, $f_{T}=8 \mathrm{GHz}, N F=1.4 \mathrm{~dB}, G=12.5 \mathrm{~dB}$, $P_{C M}=225 \mathrm{~mW}, I_{C M}=50 \mathrm{~mA}$. As to the oscillation tube $T_{1}$, the low-noise ultra-high-frequency silicon power tube 2SC3356 is selected, whose collector-junction barrier capacitance can serve as the tuned component within the oscillating circuit. Actually, the collector junction of a triode transistor is a P-N junction diode.The junction capacitance $C_{j}\left(U_{c b}\right)$ can be written as a function of the voltage between the collector and the base, $U_{c b}$ :

$$
C_{j}\left(U_{c b}\right)=\frac{C_{j}(0)}{\left(U_{c b}+\Phi\right)^{\frac{1}{2}}}
$$

Where $\Phi$ denotes the contact barrier of the semiconductor and $C_{j}(0)$ denotes the collector-junction capacitance when $U_{c b}=0$. In the present work, the silicon transistor is used, i.e., $\Phi$ varies from 0.5 to $0.7 \mathrm{~V}$. As shown in Fig. 2, $L_{1}, L_{2}, L_{3}$ and $L_{\mathrm{F}}$ are the microstrip lines.

The design of the whole VCO includes the following sections:

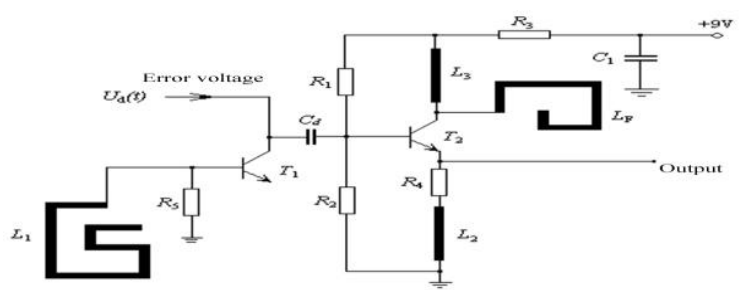

FIGURE II. CIRCUIT CONFIGURATION OF THE VCO.

\section{A. Selection of Circuit Configuration}

Generally speaking, the VCOs consisting of the variable reactance and the oscillation tube would exhibit six fundamental circuit configurations as shown in Fig. 3.

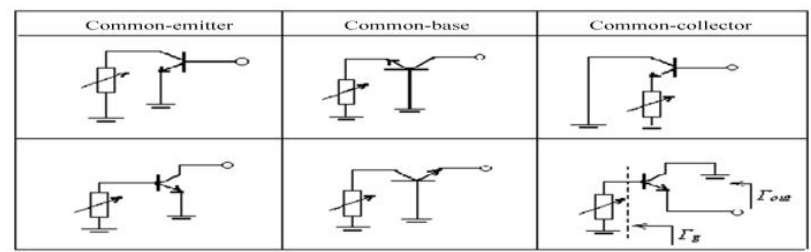

FIGURE III. FUNDAMENTAL CIRCUIT CONFIGURATIONS OF THE VCOS.

In these configurations, the oscillation can be produced only when the amplitude of the reflection coefficient of the output port $\left|\Gamma_{\text {out }}\right|$ exceeds 1 . Eq. (2) presents the relationship between the reflection coefficient of the output port $\Gamma_{\text {out }}$ and the reflection coefficient of the variable reactance, where $S_{11}$, $S_{12}, S_{21}$ and $S_{22}$ denote the small-signal $S$-parameters of the transistor in different configurations. The scattering matrix in common-emitter circuit configuration $\left[S_{e}\right]$ is often provided by the manufacturer, whereas the scattering matrices in common-base and common-collector configurations $\left[S_{b}\right]$ and $\left[S_{c}\right]$ can be acquired by means of numerical calculations using computers. According to Eq. (2), for the six circuit configurations displayed in Fig. 3, the variation curves of the output reflection coefficient phase-angle $\angle \Gamma_{g}$ can be obtained with the adoption of computer aided assembly (CAA) as displayed in Fig. 4. The curves are plotted by $\angle \Gamma_{g}$ on the horizontal axis and $\angle \Gamma_{\text {out }}$ on the vertical axis, respectively, with the unit of degree $\left(^{\circ}\right)$.

In a phase-lock loop, to accommodate the overshoot caused by the limited bandwidth in the loop amplifier with inherent frequency, we should ensure that the VCO in the loop exhibits a certain tuning bandwidth. It can be observed from Fig. 4 that by using the circuit configurations as described in (d) and (e), comparatively wider tuning ranges can be acquired provided the other conditions are identical. However, the performance is not very stable when the common-base configuration is applied, and mode hopping may occur [3]. Accordingly, the common-collector and emitter-follower output circuit is selected for the current design.

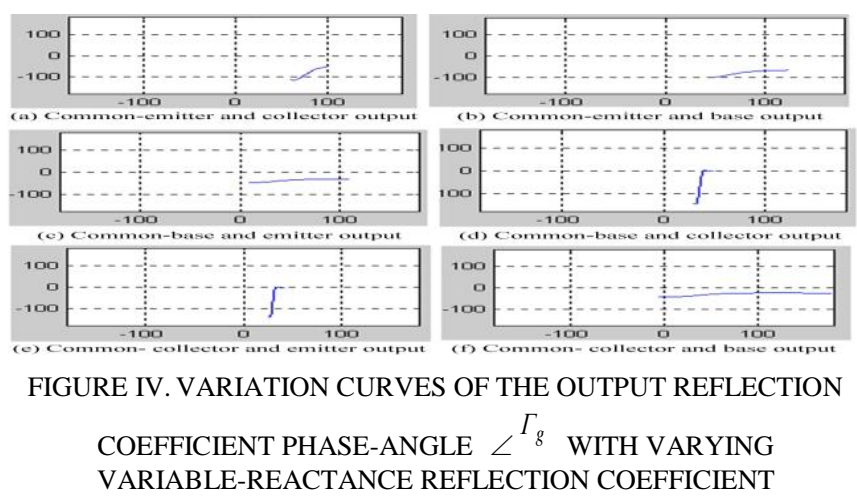

PHASE-ANGLE $\angle \Gamma_{g}$

\section{B. Main Tuned Circuit}

1) Mechanistic analysis. In accordance with Fig. 2, the radio-frequency equivalent circuit and the equivalent circuit of the resonance circuit of the VCO could be drawn as shown in Fig. 5 and Fig. 6. In Fig. 5 and Fig. 6, $L_{1}$ and $L_{\mathrm{F}}$ are acquired by using the open-circuit microstrip line with the characteristic impedance of $Z_{0} ; C_{0}$ denotes the equivalent input capacitance of the oscillation tube; $C_{d}$ denotes the coupling capacitor, and $C_{j}$ denotes the collector-junction capacitor of 2 SC3356. Aiming at improving the uniformity of the tuning sensitivity $K_{V}$ among various bands, a section of open-circuit microstrip line with the characteristic impedance of $Z_{0}$ is connected to one end of $C_{j}$ At the condition of resonance, the following expression should be satisfied 


$$
Z_{\text {in }}-j \frac{1}{\omega C_{0}}=0
$$

Where $Z_{\text {in }}$ denotes the input impedance observed from the left of the reference plane $A A^{\prime}$.

Based on transmission line theory, the equivalent impedance of the open-circuit microstrip lines can be written as: $\quad X=-j Z_{0} \cot \beta L$

Where $L$ denotes the electrical length of the open-circuit microstrip line; $\beta=2 \pi / \lambda_{g}$ and $\lambda_{g}$ denotes the wave guide wavelength of the microstrip line corresponding to the resonant frequency. Moreover, $\lambda_{g}$ exhibits the following relationship with the operating wavelength in free space, i.e., $\lambda_{g}=\frac{\lambda_{0}}{\sqrt{\varepsilon_{e}}}=\frac{c}{f \sqrt{\varepsilon_{e}}}$, where $c$ denotes the velocity of light in vacuum, whereas $\varepsilon_{e}$ denotes the effective dielectric constant of the microstrip line. As described in Ref. [4], a simple empirical formula has been deduced by M.V. Schneier,

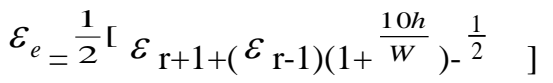

According to Fig. 6, we can obtain:

$$
Z_{\text {in }}=j\left[-\frac{1}{\omega C_{j}}-\frac{Z_{\mathrm{O}}}{\tan \left(\frac{2 \pi}{\lambda_{g}} L\right)}\right]
$$

To substitute Eq. (9) to Eq. (7), we can obtain the following expression:

$$
\frac{1}{\omega C_{j}}+\frac{z_{\mathrm{O}}}{\tan \theta}-+\frac{1}{\omega C_{\mathrm{O}}}=0
$$

where $\theta={ }^{\frac{2 \pi}{\lambda_{g}} L}=\frac{\sqrt{\varepsilon_{e}}}{c} \omega L$.

With reference to the principle of implicit differentiation, we can perform a partial differential equation on Eq. (10),

$$
\frac{\partial \omega}{\partial C_{j}}=-\frac{\omega C_{0}}{C_{j}} \cdot \frac{\tan ^{2} \theta}{C_{0} \tan ^{2} \theta+C_{j} \tan ^{2} \theta+Z_{0} \theta C_{0} C_{j}\left(1+\tan ^{2} \theta\right)}
$$

$\frac{\partial C_{j}}{\partial U}$ can be acquired by performing a partial differential equation on Eq. (6), which is then multiplied by Eq. (11). Finally, by substituting $C_{0}=-\frac{C_{j} \tan \theta}{\tan \theta+Z_{0} C_{j} \omega}$ in Eq. (11), the electrically-adjustable sensitivity can be written as:

$K_{V}=\frac{\partial \omega}{\partial U}=-\frac{0.5 \omega}{\left(U_{c b}+\Phi\right) C_{j}} \cdot \frac{\tan ^{2} \theta+Z_{0} C_{j} \omega \tan \theta}{Z_{\mathrm{o}} \theta \omega C_{j} \tan ^{2} \theta+Z_{\mathrm{o}} \omega C_{j} \tan ^{2} \theta+Z_{\mathrm{o}} \omega C_{j}(\theta-\tan \theta)}$

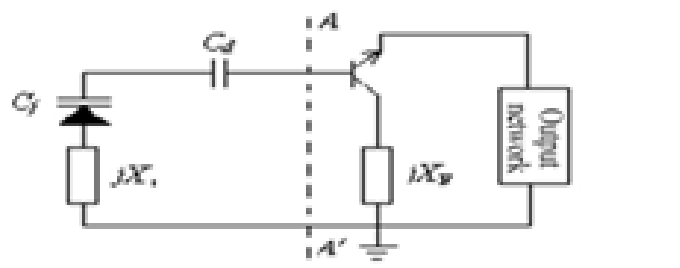

FIGURE V. RADIO-FREQUENCY CIRCUIT OF THE VCO.

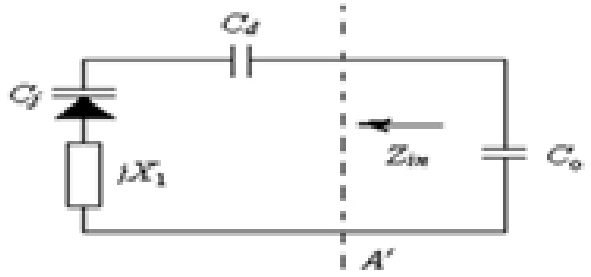

FIGURE VI. EQUIVALENT CIRCUIT OF THE RESONANCE CIRCUIT OF VCO.

2) Analysis results by simulations. As displayed in Fig. 2, L1 and LF has been implemented by the open-circuit microstrip line with the characteristic impedance of $76 \Omega$, whose length is 31 and $18 \mathrm{~mm}$, respectively. Combined with the analysis results based on the mechanistic parts, $C_{0}, C_{j}$ and the corresponding collector-junction bias-voltage values at different frequencies are obtained by means of CAA as shown in Table 1. Table 2 lists the electrically-adjustable sensitivity at different frequencies.

TABLE I. $\quad C_{0}, C_{j}$ AND THE CORRESPONDING COLLECTOR-JUNCTION

\begin{tabular}{|c|c|c|c|c|c|c|}
\hline$f /(\mathbf{G H z})$ & 1.85 & 1.90 & 1.95 & 2.00 & 2.05 & 2.10 \\
\hline $\begin{array}{c}\left(\mathbf{d} f / \mathbf{d} c_{j}\right) /(\mathbf{G H z} \cdot \mathbf{p} \\
\left.\mathbf{F}^{-1}\right)\end{array}$ & $\begin{array}{c}0.298 \\
1\end{array}$ & $\begin{array}{c}0.417 \\
4\end{array}$ & $\begin{array}{c}0.573 \\
1\end{array}$ & $\begin{array}{c}0.774 \\
0\end{array}$ & $\begin{array}{c}0.95 \\
30\end{array}$ & $\begin{array}{c}1.23 \\
00\end{array}$ \\
\hline$\left(\mathbf{d} c_{j} / \mathbf{d} U\right) /\left(\mathbf{p F} \cdot \mathbf{V}^{-}\right.$ & $\begin{array}{c}0.275 \\
0\end{array}$ & $\begin{array}{c}0.189 \\
0\end{array}$ & $\begin{array}{c}0.130 \\
0\end{array}$ & $\begin{array}{c}0.100 \\
0\end{array}$ & $\begin{array}{c}0.07 \\
80\end{array}$ & $\begin{array}{c}0.06 \\
00\end{array}$ \\
\hline $\begin{array}{c}\mathrm{d} f / \mathrm{d} U) /\left(\mathbf{G H z} \cdot \mathrm{V}^{-}\right. \\
\left.\mathbf{1}^{-}\right)\end{array}$ & $\begin{array}{c}0.082 \\
0\end{array}$ & $\begin{array}{c}0.078 \\
9\end{array}$ & $\begin{array}{c}0.074 \\
5\end{array}$ & $\begin{array}{c}0.077 \\
4\end{array}$ & $\begin{array}{c}0.07 \\
43\end{array}$ & $\begin{array}{c}0.07 \\
38\end{array}$ \\
\hline
\end{tabular}
BIAS-VOLTAGE VALUES AT DIFFERENT FREQUENCIES IN THE SIMULATIONS.

\begin{tabular}{|c|c|c|c|c|c|c|}
\hline $\begin{array}{c}\boldsymbol{F} / \\
\mathbf{G H z}\end{array}$ & 1.85 & 1.90 & 1.95 & 2.00 & 2.05 & 2.10 \\
\hline $\begin{array}{c}\boldsymbol{C}_{0} / \\
\mathbf{p F}\end{array}$ & 2.11 & 2.16 & 2.22 & 2.29 & 2.36 & 2.43 \\
\hline $\boldsymbol{C}_{j / \mathbf{p F}}$ & 1.81 & 1.69 & 1.59 & 1.50 & 1.42 & 1.36 \\
\hline $\begin{array}{c}U_{c b} / \\
\mathbf{V}\end{array}$ & 1.19 & 1.84 & 2.61 & 3.42 & 4.48 & 5.93 \\
\hline
\end{tabular}

TABLE II. ELECTRICALLY-ADJUSTABLE SENSITIVITY AT DIFFERENT FREQUENCIES IN THE SIMULATIONS.

\begin{tabular}{|c|c|c|c|c|c|c|}
\hline $\mathbf{f}(\mathbf{G H z})$ & 1.85 & 1.90 & 1.95 & 2.00 & 2.05 & 2.10 \\
\hline $\begin{array}{c}\left(\mathbf{d} f / \mathbf{d} c_{j}\right) /(\mathbf{G H z} \cdot \mathbf{p} \\
\left.\mathbf{F}^{-1}\right)\end{array}$ & $\begin{array}{c}0.303 \\
8\end{array}$ & $\begin{array}{c}0.417 \\
5\end{array}$ & $\begin{array}{c}0.544 \\
6\end{array}$ & $\begin{array}{c}0.657 \\
0\end{array}$ & $\begin{array}{c}0.774 \\
4\end{array}$ & $\begin{array}{c}0.923 \\
3\end{array}$ \\
\hline$\left.\left(\mathbf{d} c_{j} / \mathbf{d} U\right)_{-1}^{-1}\right)(\mathbf{p F} \cdot \mathrm{V}$ & $\begin{array}{c}0.275 \\
0\end{array}$ & $\begin{array}{c}0.189 \\
0\end{array}$ & $\begin{array}{c}0.130 \\
0\end{array}$ & $\begin{array}{c}0.100 \\
0\end{array}$ & $\begin{array}{c}0.078 \\
0\end{array}$ & $\begin{array}{c}0.060 \\
0\end{array}$ \\
\hline $\begin{array}{c}\mathrm{d} f / \mathrm{d} U) /(\mathbf{G H z} \cdot \mathrm{V} \\
-1)\end{array}$ & $\begin{array}{c}0.083 \\
5\end{array}$ & $\begin{array}{l}0.078 \\
9\end{array}$ & $\begin{array}{c}0.070 \\
8\end{array}$ & $\begin{array}{c}0.065 \\
7\end{array}$ & $\begin{array}{c}0.060 \\
4\end{array}$ & $\begin{array}{c}0.055 \\
4\end{array}$ \\
\hline
\end{tabular}

TABLE III. ELECTRICALLY-ADJUSTABLE SENSITIVITY AT DIFFERENT FREQUENCIES IN THE SIMULATIONS, WITH L1 IMPLEMENTED BY THE LUMPED PARAMETERS.

The CAA analysis results indicate that $K_{V}$ gradually decreases from the low-frequency band towards the high-frequency band within the tuning range of the VCO. When the open-circuit microstrip line with the length of $\theta$ is 
connected, the variation tends to become more complex, rather than the original monotone-decreasing trend as shown in Table 3. As indicated in Eq. (12), the electrical length of the open-circuit micro-strip line $\theta$ imposes a crucial effect on affecting the variation tendency of $K_{V}$. Due to different bias voltage values, $\theta$ varies correspondingly. To be specific, if the numerator exerts an increasing effect on $K_{V}$, the denominator will exert a decreasing effect on $K_{V}$. Otherwise, if the numerator exerts a decreasing effect on $K_{V}$, the denominator will then exert an increasing effect on $K_{V}$. Accordingly, the variation tendency that $K_{V}$ varies monotonously towards a specific direction in the tuning range can be hampered, i.e., the uniformity of the electrically-adjustable sensitivity in the tuning band can be improved. These conclusions can be verified by the analysis results as listed in Table 2 and Table 3 using CAA.

\section{Equivalent Phase-Shift Contour}

In a monolithic microwave integrated circuit (MMIC), the external capacitor is not suitable when choosing a feedback capacitor, due to the incompatibility between the external capacitor with a discrete structure and the microstrip film. In practice, we figure out that making a connection between the electrode tip that is not directly connected to the tuned circuit and the reactance should be an effective approach. By selecting microstrip lines with different lengths and characteristic impedance as the adjustable termination, the optimum negative conductance could be acquired. The adjustment of the electrical length of the terminated microstrip line can be regarded as the equivalent adjustment of the amount of phase shift or the adjustment of the feedback quantity between electrodes. This adjustment contributes to initiating an oscillation, changing the power and fine tuning the frequency, all of which has been proved effective in circuit debugging.

\section{PREPARATION OF THE VCO AND RELATED SIMULATION RESULTS}

The double-face copper-clad composite substrate with the depth of $1 \mathrm{~mm}$, the ...of $0.18 \mathrm{~mm}$ and the ...of 4.5 was selected. $L_{1}$ and $L_{F}$ were implemented through the open-circuit microstrip line with the width of $0.8 \mathrm{~mm}$, and the microstrip lines with the width of $2.2 \mathrm{~mm}$ were selected as the $50-\Omega$ transmission line. In order to accomplish the circuit's miniaturization, the effective lengths of the open-circuit microstrip lines in both tuning network and feedback network were made into U-shape and $\mathrm{W}$-shape. At different collector-junction bias voltages $U_{c b}$, the output frequency $f$ as well as the input frequency $P$ of the prepared VCO were measured, with the results as shown in Table 4. Table 5 lists the comparison results between the simulations results and the measured values of the collector-junction bias voltage $U_{c b}$ at the same frequencies.
TABLE IV. VARIATIONS OF THE OUTPUT FREQUENCY AND THE INPUT FREQUENCY OF THE PREPARED VCO WITH VARYING COLLECTOR-JUNCTION BIAS VOLTAGE $U_{c b}$

\begin{tabular}{|c|c|c|c|c|c|c|}
\hline $\boldsymbol{f} / \mathbf{G H z}$ & 1.85 & 1.90 & 1.95 & 2.00 & 2.05 & 2.10 \\
\hline $\boldsymbol{P} / \mathbf{m W}$ & 5.0 & 5.3 & 5.5 & 5.1 & 4.6 & 4.2 \\
\hline$U_{c b} / \mathbf{V}$ & 1.4 & 2.0 & 2.8 & 3.4 & 4.2 & 5.5 \\
\hline
\end{tabular}

TABLE V. COMPARISONS BETWEEN THE MEASURED VALUES AND THE CALCULATION VALUES OF THE ELECTRICALLY ADJUSTABLE PERFORMANCE.

\begin{tabular}{|c|c|c|c|c|c|c|}
\hline $\begin{array}{c}\text { Oscillation frequency }(f / \\
\text { GHz) }\end{array}$ & $\begin{array}{c}1.8 \\
5\end{array}$ & $\begin{array}{c}1.9 \\
0\end{array}$ & $\begin{array}{c}1.9 \\
5\end{array}$ & $\begin{array}{c}2.0 \\
0\end{array}$ & $\begin{array}{c}2.0 \\
5\end{array}$ & $\begin{array}{c}2.1 \\
0\end{array}$ \\
\hline $\begin{array}{c}\text { Measured bias voltage }\left(\boldsymbol{U}_{c b}\right. \\
/ \mathbf{V})\end{array}$ & 1.4 & 2.0 & 2.8 & 3.4 & 4.2 & 5.5 \\
\hline $\begin{array}{c}\text { Calculated bias } \\
\text { voltage }\left(U_{c b} / \mathrm{V}\right)\end{array}$ & $\begin{array}{c}1.1 \\
9\end{array}$ & $\begin{array}{c}1.8 \\
4\end{array}$ & $\begin{array}{c}2.6 \\
1\end{array}$ & $\begin{array}{c}3.4 \\
2\end{array}$ & $\begin{array}{c}4.4 \\
8\end{array}$ & $\begin{array}{c}5.9 \\
3\end{array}$ \\
\hline
\end{tabular}

\section{CONCLUSIONS}

It can be concluded from the above-described simulation analyses that the output tuning range of the prepared voltage-controlled oscillator (VCO) varies from 1.85 to 2.1 $\mathrm{GHz}$, with a favorable power flatness in the band $(4.2 \sim 5.5$ $\mathrm{mW})$. The results suggest that it is an appropriate and effective approach to design and analyze the VCO by means of computer aided design (CAD) and computer aided assembly (CAA). Due to the access of open-circuit microstrip lines in the tuned loop, the uniformity of the electrically adjustable sensitivity in the tuning band has been improved. The adjustment of the length of the open-circuit microstrip lines terminated to the collector, or in other words, the adjustment of the feedback quantity between the electrodes, can contribute to the oscillation startup condition and ultimately achieving the goal of fine adjustments of the power and center frequency.

\section{REFERENCES}

[1] Y. C. Fei. Microwave Solid-state Frequency Source, Beijing: National Defense Industry Press, pp. 19-25, 1992.

[2] Y. C. Fei. \& S. W. Chen. Microwave Solid-state Frequency Source: Theory, Design and Application, Beijing: National Defense Industry Press, pp. 113-221, 1996.

[3] W. H.Chen \& Y. M. Li .Microwave Oscillator, Beijing: Posts \& Telecom Press, pp. 32-38, 1984.

[4] S. S. Zhong. Theory and Application of Microstrip Antenna, Xi'an: Xidian University Press, pp. 94-96, 1990.

[5] J. W. Ye. \& R. H. Qiu. \& J. F. Ye. Electronic Circuits of Communication: Principle and Simulation Design, Electronic Industry Press, pp. 142-145, 2012. 